\title{
Nichtbegründetes Nichtannehmen
}

\section{§ 93d Abs. 1 S. 3 BVerfGG als verfassungsprozessualer Irrweg \\ Carsten Bäcker $\bowtie$}

I. Entlastung statt Begründung

1. Die frühe Kritik

a) Abschaffung der Verfas-

sungsbeschwerde........... 482

b) Zurück zur Aristokratie..... 484

2. Die Rechtfertigung............ 485

a) Nichtbegründung als Entlastung.

b) Verfassungsbeschwerde als außerordentlicher Rechtsbehelf.

3. Grundgesetzlicher Begründungszwang gerichtlicher Entscheidungen?.
II. Begründung als Legitimation 491

1. Entscheidungsgründe im Prozeßrecht

2. Begründungspflicht des Bundesverfassungsgerichts.

a) Das Bundesverfassungsgericht als Letztinterpret..... 495

b) Begründungslosigkeit als Legitimitätsverlust .

III. Ergebnis . 499

Das 5. Gesetz zur Änderung des Gesetzes über das Bundesverfassungsgericht vom 2. August $1993^{1}$ schrieb dem Verfassungsprozeßrecht Neuerungen ein, die bis heute Bestand haben. Ein Kern der Neuerungen ${ }^{2}$ war die Neuregelung des 1963 eingerichteten und seitdem skeptisch begleiteten ${ }^{3}$ Annahmeverfahrens von Verfassungsbeschwerden. Die Neugestaltung des Annahmeverfahrens im Jahr 1993 schaffte die im Gesetzgebungsprozeß kontrovers diskutierte ${ }^{4}$ und vom Gericht drängend eingeforderte $^{5}$ Möglichkeit, die Annahme einer Verfassungsbeschwerde gänzlich ohne Begründung abzulehnen.

Dr. Carsten Bäcker ist wissenschaftlicher Mitarbeiter am Hermann Kantorowicz-Institut für juristische Grundlagenforschung der Rechtswissenschaftlichen Fakultät der Christian-Albrechts-Universität zu Kiel.

1 BGBl. I, S. 1442-1445.

2 Vgl. BT-Dr. 12/3628, S. 7-14.

3 Vgl. etwa F. Klein, Die dritte Novelle zum Bundesverfassungsgerichtsgesetz vom 3. August 1963, DVBl. 1964, S. 89-92, 90 f.; K. Vogel, „Offensichtlich unbegründet“, JZ 1969, S. 801; W. Speckmann, Zur Praxis der Dreier-Ausschüsse nach $\mathbb{S} 93$ a BVerfGG, JZ 1970, S. 213 f.; R. Zuck, Zur Reform des Verfahrens vor dem Bundesverfassungsgericht, ZRP 1973, S. 233-239 (238 f.); K. Löw, Dauerproblem Verfassungsbeschwerde, DVBl. 1973, S. 941-944; breiter angelegt H. F. Zacher, Die Selektion der Verfassungsbeschwerden - Die Siebfunktion der Vorprüfung, des Erfordernisses der Rechtswegerschöpfung und des Kriteriums der unmittelbaren und gegenwärtigen Betroffenheit des Beschwerdeführers, in: C. Starck (Hrsg.), Bundesverfassungsgericht und Grundgesetz. Festgabe aus Anlaß des 25jährigen Bestehens des Bundesverfassungsgerichts, Tübingen: Mohr Siebeck 1976, S. 396-431; C. Sailer, Verfassungsbeschwerde im Zwielicht, ZRP 1977, S. 303-310, 304 f.; W. Graf Vitzthum, Das Vorprüfungsverfahren für Verfassungsbeschwerden, in: G. Püttner (Hrsg.), Festschrift für Otto Bachof zum 70. Geburtstag, München: Beck 1984, S. 293-320; ferner die Stellungnahme des Verfassungsrechtsausschusses zum Entwurf des Vierten Gesetzes zur Änderung des Gesetzes über das Bundesverfassungsgericht, AnwBl 1970, S. $127 \mathrm{f}$.

4 Vgl. die Bedenken des Bundesrates, BT-Dr. 12/3628, S. 16.

5 Vgl. etwa die Schilderung der Anhörung des Präsidenten und des Vizepräsidenten des Bundesverfassungsgerichts vor dem Rechtsausschuß, BT-Dr. IV/1366, S. 1 f. 
Diese Möglichkeit der nichtbegründeten Nichtannahme von Verfassungsbeschwerden hat Ausnahmecharakter. Sie erlaubt eine Abweichung von der generellen Begründungspflicht für gerichtliche Entscheidungen, in $\$ 30$ Abs. 1 S. 2 BVerfGG dem Verfassungsprozeßrecht eingeschrieben und ansonsten nur durch $\mathbb{2} 24$ BVerfGG durchbrochen. ${ }^{6}$ Der 1993 eingeführte $\mathbb{\$}$ 93d Abs. 1 BVerfGG lautet auch in der aktuellen Fassung:

Die Entscheidung nach $\ 93 b$ [Ablehnung oder Annahme einer Verfassungsbeschwerde durch die Kammer] und \$93c [Stattgabe einer Verfassungsbeschwerde durch die Kammer] ergeht ohne mündliche Verhandlung. Sie ist unanfechtbar. Die Ablebnung der Annahme der Verfassungsbeschwerde bedarf keiner Begründung.

Kritik an dieser exzeptionellen Regelung des Verfassungsprozeßrechts wurde insbesondere im Zuge der Gesetzesänderung vorgetragen. ${ }^{7}$ Sie ist noch immer nicht verstummt. Inwiefern sie heute, soweit sie die Nichtbegründung betrifft, noch berechtigt vorgebracht werden kann, wird im vorliegenden Beitrag überprüft. Dabei wird sich zeigen, daß die Diskussion über die Frage der Verfassungsmäßigkeit der nichtbegründeten Nichtannahme hinausgehen muß. Es geht letztlich um die Legitimität der Verfassungsgerichtsbarkeit - und damit um ein metarechtliches Problem.

\section{Entlastung statt Begründung}

Da sich die für die vorliegende Untersuchung interessante Vorschrift bis heute nicht geändert hat, könnte von der unterbliebenen Änderung der Vorschrift auf eine Überholung der Kritik geschlossen werden, nicht auf eine ungeminderte Berechtigung. Doch wäre das voreilig, denn im Kern erweist sich die frühe Kritik als noch immer aktuell.

\section{Die frühe Kritik}

Die Kritiker, vornehmlich aus den Kreisen der beschwerdeführenden Seite, sahen in den Änderungen einen grundlegenden Wandel des Verfassungsbeschwerdeverfahrens; ein Wandel, der dramatische Konsequenzen nach sich ziehen müsse. Diese Konsequenzen bestünden, so Rüdiger Zuck, in der faktischen Abschaffung der Verfassungsbeschwerde; für Hermann Kroitzsch glich der Wegfall der Begründungspflicht gar einem Rückfall in eine judikative Aristokratie.

\section{a) Abschaffung der Verfassungsbeschwerde}

Zuck, Rechtsanwalt und Coautor des Beck'schen Standardkurzkommentars zum BVerfGG, formulierte seine Kritik erstmals unmittelbar nach der Bekanntgabe des

6 Nach $\$ 24$ BVerfGG kann das Gericht einen Antrag ohne Begründung verwerfen, wenn es dem Antragsteller zuvor ein Belehrungsschreiben übermittelt hat, in dem es seine Bedenken gegen die Zulässigkeit oder Begründetheit des Antrags schildert. In diesem Belehrungsschreiben läßt sich eine vorweggenommene Begründung der Ablehnung erblicken.

7 Vgl. neben den sogleich näher betrachteten Stimmen auch E. Klein, Konzentration durch Entlastung? Das Fünfte Gesetz zur Änderung des Gesetzes über das Bundesverfassungsgericht, NJW 1993, S. 2073-2077. 
5. Änderungsgesetzes. Das dem Gesetzgeber unterstellte Ziel, „jedermann die Verfassungsbeschwerde von Verfassungs wegen zu gewährleisten, sie aber so gut wie niemandem praktisch zu geben “, sei nunmehr wegen kaum zu erfüllender oder vage formulierter Annahmevoraussetzungen „endlich erreicht“; die „faktische Abschaffung der Verfassungsbeschwerde" vollkommen. Auch eine Kontrolle der Beachtung der Annahmevoraussetzungen seitens des Gerichts durch die Rechtswissenschaft oder die Öffentlichkeit könne es schlicht nicht mehr geben, weil „die Nichtannahmeentscheidung $[\ldots]$ nicht zu begründen “ ist. ${ }^{8}$

Der guten Absicht des Gesetzgebers, dem Bundesverfassungsgericht mit der Neure-

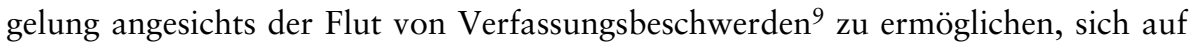
die wirklich bedeutsamen Verfahren diesseits und jenseits der Verfassungsbeschwerden zu konzentrieren, hält Zuck entgegen, daß das Bundesverfassungsgericht, notfalls unter Aufstockung des Personals, ${ }^{10}$ „imstande sein “ müsse, „seine Aufgaben nach Art. 93 I Nr. 4a GG“ zu erfüllen. ${ }^{11}$ Es sei, so Zuck in formelhafter Wendung, „nicht Aufgabe des Gerichts, sich zu entlasten, sondern [...] seinen Verfassungsauftrag zu erfüllen “. ${ }^{12}$

Der Verzicht auf eine „Begründungspflicht bei Nichtannahmebeschlüssen“ sei jedenfalls „rechtsstaatswidrig “ und überdies „,europarechtsfeindlich “. ${ }^{13}$ Gerade in der Frage, ob die Annahme einer Verfassungsbeschwerde angezeigt wäre, in deren Beantwortung es einen „richterlichen Beurteilungsspielraum “ der Verfahrenseröffnung gebe, sei es rechtsstaatlich schlechterdings nicht tragbar, den „Bürger zu einem bloßen Verfahrensobjekt" zu machen. Europarechtlich sei zu beachten, daß das Verfassungsbeschwerdeverfahren vor dem Bundesverfassungsgericht „angesichts der Möglichkeit der Menschenrechtsbeschwerde" nicht die letzte Instanz sei. Eine Begründung der Nichtannahmeentscheidung sei aber Bedingung ihrer Überprüfbarkeit. ${ }^{14}$

Soweit manche Nichtannahmeentscheidungen allein mit dem Hinweis versehen würden, daß keine der gesetzlichen Annahmegründe unter Wiederholung derselben vor-

8 R. Zuck, Der Zugang zum BVerfG: Was läßt das 5. Änderungsgesetz zum Gesetz über das BVerfG von der Verfassungsbeschwerde noch übrig?, NJW 1993, S. 2641-2646, 2641.

9 Die Bewältigung dieser Flut war stets Motivation der Novellen des BVerfGG. Ebenso war die Erhöhung der Zugangshürden stets Anlaß zur Kritik, vgl. nur B. Schlink, Zugangshürden im Verfassungsbeschwerdeverfahren, NJW 1984, S. 89-94, bei Anerkennung der „Notwendigkeit eines Vorprüfungsund Annahmeverfahrens“, S. 90. Besonders in der Kritik stand die „abgekürzte Begründung der Nichtannahme, die sich in der Sache als Entscheidung über die Verfassungsbeschwerde selbst" darstelle, vgl. C. Gusy, Die Verfassungsbeschwerde: Voraussetzung und Verfahren, Heidelberg 1988, S. 177.

10 Für Zuck, Zugang (Fn. 8), S. 2642, ist es dabei nicht mit einer Ausweitung des Mitarbeiterstabs der Verfassungsrichter getan, da der Richter seine rechtsprechende Gewalt nicht übertragen könne. Ihm schwebt ein Kammersystem vor, in dem ein Verfassungsrichter den Vorsitz hat und zwei bloße Kammerrichter mitwirken.

11 Zuck, Zugang (Fn. 8), S. 2642.

12 Zuck, Zugang (Fn. 8), S. 2646.

13 Zuck, Zugang (Fn. 8), S. 2646.

14 Zuck, Zugang (Fn. 8), S. 2646. 
lägen, sei darin eben keine Begründung, sondern eine nur „wenig vertrauensbildende Maßnahme“ zu sehen. ${ }^{15}$ Insgesamt befindet Zuck „die Aufnahme einiger begründender Sätze auch unter Entlastungsaspekten“ für durchaus „vertretbar“, ${ }^{16}$ dem Gericht also zumutbar.

In einem späteren Kommentar fügt Zuck hinzu, daß auch eine Nichtannahmeentscheidung „vom Berichterstatter (häufig umfangreich) votiert und von den übrigen Kammermitgliedern mitgetragen werden“ müsse. Die vermeintliche „Arbeitsentlastung “ durch eine Nichtbegründung bestehe dann nur noch darin, „daß aus dem Votum keine tragenden Gründe für die Ablehnungsentscheidung herausdestilliert werden müssen, außerdem in der Reduzierung von Schreibarbeit". ${ }^{17}$

Zuck beschließt seine Kritik mit einer schmerzlichen Vermutung: „Gewichtiger“ als die Arbeitsentlastung sei „wohl die subjektive Entlastung: Wer seine Gründe nicht bekanntgibt, nimmt in der Sache nicht Stellung, und übernimmt deshalb auch keine Verantwortung “. Für den Bürger bleibe von seiner Verfassungsbeschwerde im Fall der nichtbegründeten Nichtannahme am Ende des Tages nur die „kalte[...] Reaktion des leeren Blattes“. $\$ 93 \mathrm{~d}$ Abs. 1 S. 3 BVerfGG zeitige so „menschenverachtende[...] Auswirkungen “. ${ }^{18}$

\section{b) Zurück zur Aristokratie}

Kroitzsch, Rechtsanwalt beim BGH, wendete sich, ähnlich vehement wie Zuck, 1994 gegen den Wegfall der Begründungspflicht. Er sieht in ihr den Beleg für den „Wandel der Staatsform der Bundesrepublik“, von einem demokratischen und sozialen Bundesstaat hin zu einer Aristokratie. ${ }^{19}$ Er geht dabei von grundsätzlichen Überlegungen zur Begründungspflicht aus. Die Begründungspflicht von Gerichtsurteilen sieht er als Ausdruck des verfassungsrechtlichen Öffentlichkeitsprinzips, das im demokratischen Parlamentarismus dem Zweck der Kontrolle der vom Volk ausgehenden Staatsgewalt durch das Volk diene. ${ }^{20}$ Zusammenfassend führt er aus: ${ }^{21}$

15 Zuck, Zugang (Fn. 8), S. 2646.

16 Zuck stimmt hier E. Klein, Entlastung (Fn. 7), S. 2075, zu, für den die Begründung der Ablehnungsentscheidung angesichts des vorliegenden Votums „kein erheblicher Mehraufwand“ sei. - Schärfer formuliert den gleichen Befund E. Schneider, Rechtsstaat ohne Begründungszwang?, ZIP 1996, S. 487: „Für jeden halbwegs intelligenten Juristen ist es [...] keine ins Gewicht fallende Arbeit, zwei oder drei Kernsätze zu diktieren, mit denen er seine grundsätzlichen Erwägungen verlautbart. Notfalls kann der Entscheidungsverfasser auf seine Notizen oder sein Votum zurückgreifen, vorausgesetzt allerdings, er hat den Fall durchdacht".

17 R. Zuck, Vom Winde verweht: $\$$ 93d BVerfGG und menschliche Schicksale, NJW 1997, S. 29 f., 29.

18 Zuck, Winde (Fn. 17), S. 30.

19 H. Kroitzsch, Wegfall der Begründungspflicht - Wandel der Staatsform der Bundesrepublik, NJW 1994, S. 1032-1035, 1032.

20 Vgl. Kroitzsch, Wegfall (Fn. 19), S. 1032 f. - Ähnlich Schneider, Begründungszwang (Fn. 16), S. 487: „Der rechtsstaatlich unverzichtbaren externen Kontrolle durch den Rechtsunterworfenen wird kaum noch Bedeutung beigemessen“.

21 Kroitzsch, Wegfall (Fn. 19), S. 1033. 
Begründungspflichten stehen damit nicht nur im Geiste der Rechtsstaatlichkeit, sondern auch der demokratischen Legitimität, insbesondere der Akzeptanz und der Transparenz staatlicher Entscheidungen.

Die Einführung der Möglichkeit der nichtbegründeten Nichtannahme durch $\$ 93 \mathrm{~d}$ Abs. 1 BVerfGG fiel, so Kroitzsch, ausgerechnet in eine Zeit, in der den Bürgern der neuen Länder erstmals „die Verfassungsbeschwerde als letzte Möglichkeit eines umfassenden Rechtsschutzes“ zur Verfügung stand. ${ }^{22}$ Auf vom Bundesrat geäußerte, entsprechende Bedenken hin habe die Bundesregierung, was zutrifft, auf die Zumutbarkeit für den betroffenen Bürger verwiesen, auch im Falle des Absehens von einer Begründung „dies als abschließende Äußerung eines obersten Verfassungsorgans in einem außerordentlichen Rechtsbehelfsverfahren zu akzeptieren ". ${ }^{23}$ In dieser als „großer Barschheit“ ${ }^{24}$ bewerteten Äußerung erblickt Kroitzsch den Rückfall in eine judikative Aristokratie als „Herrschaft der Besten, Klügsten und Edelsten“. 25

Eine solche Freiheit der Bundesverfassungsrichter dürfe es, so mahnte Kroitzsch abschließend, nicht geben. Denn jeder Bürger habe „einen Anspruch darauf, daß er die Entscheidungsgründe [...] im Verfahren der Verfassungsbeschwerde erfährt “. ${ }^{26} \mathrm{Oh}$ ne Angabe von Gründen seien die Verfassungsrichter „jeder demokratischen Kontrolle“ ${ }^{\text {"27 }}$ enthoben.

\section{Die Rechtfertigung}

Auch wenn die düsteren Prognosen der frühen Kritiker nicht eingetroffen sind, es insbesondere noch immer, wenn auch nur zu einem geringen Anteil, ${ }^{28}$ erfolgreiche Verfassungsbeschwerden gibt und die grundgesetzliche Demokratie noch immer keiner judikativen Aristokratie gewichen ist, so ist doch bis heute eines zu konstatieren: Der 1993 eingeführte, restlose Fortfall der Begründungspflicht für Nichtannahmen ist eigentümlich radikal. ${ }^{29}$ Jede nichtbegründete Entscheidung steht im Verdacht der

22 Kroitzsch, Wegfall (Fn. 19), S. 1034.

23 Nachzulesen in BT-Dr. 12/3628, S. 18.

24 E. Klein, Entlastung (Fn. 7), S. 2075, dort Fn. 25.

25 Kroitzsch, Wegfall (Fn. 19), S. 1035.

26 Kroitzsch, Wegfall (Fn. 19), S. 1035. Die Grundlage für diesen Begründungsanspruch erblickt Kroitzsch (wohl) im Verfassungsgrundsatz des demokratischen Bundesstaats.

27 Kroitzsch, Wegfall (Fn. 19), S. 1035.

28 Nach der amtlichen Statistik liegt die Erfolgsquote von Verfassungsbeschwerden bei 2,4\%, vgl. https:// www.bundesverfassungsgericht.de/organisation/gb2013/A-I-1.html.

29 E. Klein, Entlastung (Fn. 7), S. 2075: „höchst ungut“. 
Beliebigkeit. ${ }^{30}$ Dieser Verdacht läßt sich wohl nur entkräften, wenn die nichtannehmenden Kammern im Sinne der vorherigen gesetzlichen Fassungen ${ }^{31}$ einen Hinweis auf den „für die Ablehnung maßgeblichen rechtlichen Gesichtspunkt“ geben würden, wenn auch nur im Rahmen einer summarischen Prüfung. ${ }^{32}$

\section{a) Nichtbegründung als Entlastung}

Befürworter der Begründungslosigkeit, vornehmlich aus den Kreisen der beschwerdeprüfenden Seite, halten auch einem derart eingeschränkten Begründungserfordernis entgegen, daß die Abfassung selbst nur einiger weniger begründender Sätze zu

30 Den Vorwurf der Beliebigkeit erhebt etwa H. Sendler, Kammermusik II - Kammerrechtsprechung und gesetzlicher Richter, NJW 1995, S. 3291 f., 3292, der mit Bezug auf ohne Begründung nicht angenommene Verfassungsbeschwerden ausführt: „Die Spannbreite der Alternativen jedenfalls ist erstaunlich und muß erschrecken: offensichtlich begründete Verfassungsbeschwerden einerseits, deren begründungslose Nichtannahme andererseits. Beliebigkeit? Beliebigkeit!“; zustimmend U. Kakeldey, Recht und billig oder recht und beliebig? Eine Anmerkung zu Art. 20 Abs. 3 GG, AnwBl. 1998, S. 38 f., 39; ferner Zuck, Winde (Fn. 17), S. 29: „\$93 d I 3 BVerfGG [...] stellt die Beifügung einer Begründung $[\ldots]$ in das Belieben des Gerichts. Ich vermeide das Wort ,Ermessen', weil dann die Begründungsmodalitäten allgemeiner rechtsstaatlicher Kontrolle unterlägen. Das ist hier jedoch nicht der Fall““. - Nur etwas zurückhaltender M. Bruns, Art. 6 I GG und gesetzliche Regelungen für gleichgeschlechtliche Lebensgemeinschaften, ZRP 1996, S. 6-10, 10: Das Bundesverfassungsgericht könne „sogar begründete Verfassungsbeschwerden fast nach Belieben annehmen oder ablehnen“.

31 Die vorherigen Fassungen sahen alle ein (moderates) Begründungserfordernis für die Nichtannahme einer Verfassungsbeschwerde vor. So enthielt $\$ 91$ a BVerfGG in der Fassung des Ersten Gesetzes zur Änderung des Gesetzes über das Bundesverfassungsgericht vom 21. Juli 1956 (BGBl. I, S. 662-665) für die Begründung einer Ablehnung der Annahme einer Verfassungsbeschwerde, die nur einstimmig von dem aus drei Verfassungsrichtern bestehenden Vorprüfungsausschuß beschlossen werden konnte, einen Verweis auf $\$ 24$ S. 2 BVerfGG. Danach bedurfte die Nichtannahme (nur) dann keiner Begründung, ,wenn der Antragsteller vorher auf die Bedenken gegen die Zulässigkeit oder Begründetheit seines Antrags hingewiesen worden ist". Diesen Bedenken ließen sich die Gründe der Ablehnung entnehmen, sie war insofern wenigstens mittelbar begründet. Das dritte Gesetz zur Änderung des Gesetzes über das Bundesverfassungsgericht vom 3. August 1963 (BGBl. I, S. 589 f.) ließ $\$ 91$ a entfallen, und regelte in $\$ 93 a$ Abs. 4 BVerfGG, daß der Beschluß des Dreierausschusses oder des Senats über die Nichtannahme ,unter Hinweis auf den für die Ablehnung [...] maßgeblichen rechtlichen Gesichtspunkt" mitzuteilen ist. Erst Jahre später hob das Neunzehnte Gesetz zur Änderung des Grundgesetzes vom 29. Januar 1969 (BGBl. I, S. 97) die Verfassungsbeschwerde in den Rang eines verfassungsrechtlich vorgesehen Verfahrens, Art. 93 Abs. 1 Nr. 4a GG. Zugleich wurde Art. 94 Abs. 2 GG ein Satz angefügt, nach dem der Verfassungsbeschwerde ein besonderes Annahmeverfahren per Gesetz vorgeschaltet werden kann. Das Vierte Gesetz zur Änderung des Gesetzes über das Bundesverfassungsgericht vom 21. Dezember 1970 (BGBl. I, S. 1765-1768) erweiterte die zulässigen Gründe für eine Ablehnung der Annahme einer Verfassungsbeschwerde, änderte aber nichts an dem Begründungserfordernis. Auch das Gesetz zur Änderung des Gesetzes über das Bundesverfassungsgericht und das Deutsche Richtergesetz vom 12. Dezember 1985 (BGBl. I, S. 2226-2228) regelte das Begründungsbedürfnis lediglich an anderer Stelle (\$93b Abs. 3 S. 2 BVerfGG), und brachte ansonsten abermals eine Erweiterung der möglichen Gründe für eine Ablehnung der Annahme der Verfassungsbeschwerde durch die Kammer ( $\$ 93 b$ Abs. 1 BVerfGG).

32 Ein aktuelles Beispiel für eine diesen Anforderungen vollumfänglich entsprechende Begründung einer Nichtannahme ist der Beschluß der 3. Kammer des Ersten Senats vom 3. September 2014, 1 BvR $3353 / 13$. 
einer deutlich geringeren Entlastung des Gerichts führen würde. ${ }^{33}$ Denn die Formulierung der Beschlußgründe müsse, trotz des Vorliegens eines Votums, worauf Karin Graßhof im Beck'schen Großkommentar ${ }^{34}$ und Franz Schemmer im Mitarbeiterkommentar hinweisen, „sorgfältig erfolgen, damit Unklarheiten, Missverständnisse oder Missdeutungen ausgeschlossen “ 35 werden könnten. Auch könnten „die beiden anderen Richter der Kammer unterschiedliche Vorstellungen über die Art und den Umfang der Begründung der Nichtannahme haben“, weswegen der „Verzicht auf die Begründung“ den „Zwang zum Konsens“ verringere, und sogar "gelegentlich Voraussetzung dafür“ sei, daß „überhaupt einstimmig - bezogen auf das Ergebnis entschieden" werde. ${ }^{36}$

Diese Argumentation macht, erstens, geltend, daß eine Bekanntgabe der Entscheidungsgründe sehr sorgfältig erfolgen müsse, weil sie präjudizielle Wirkung entfalten könnte, und, zweitens, es wenigstens in einigen Fällen keine für den Nichtannahmebeschluß der Kammer gem. $\$ 93 \mathrm{~d}$ Abs. 3 BVerfGG erforderliche Einstimmigkeit geben würde, wenn die Nichtannahme mit Gründen zu versehen wäre. Beides ist im Ergebnis, auch wenn damit ohne Frage eine geringere Entlastung des Gerichts einhergeht, Wasser auf die Mühlen der frühen Kritiker. Es geht ihnen um das Vertrauen des Bürgers in eine sorgfältige Auseinandersetzung mit den eingegangenen Verfassungsbeschwerden; hinter den Kulissen wird das obligatorische Votum des Mitarbeiters diese Sorgfalt ohnehin bereits an den Tag gelegt haben. Die Bekanntgabe der votierten Nichtannahmegründe mit präjudizieller Wirkung würde dann aber ein Mehr an Rechtssicherheit im Sinne präjudizieller Bindung bringen. Jede ausbleibende Nichtannahme hätte überdies die,

33 Vgl. Graßhof, $\mathbb{S}$ 93d, in: Bundesverfassungsgerichtsgesetz. Kommentar, begr. v. T. Maunz, 20. Lfg. München 2001 (Stand: Februar 2013, 40. Lfg.), Rn. 5: „Man ging davon aus, dass nach der Neuregelung des Annahmeverfahrens eine Begründung des Nichtannahmebeschlusses auszuführen hätte, dass die Annahmegründe der Buchstaben a und b des $\mathbb{9} 93 \mathrm{a}$ Abs. 2 nicht vorliegen, während nach bis dahin geltender Regelung die Voraussetzungen für das Vorliegen der Ablehnungsgründe darzulegen waren. Wäre nach neuem Recht eine Begründung geboten, so seien umfassendere Ausführungen als vorher erforderlich. Das liefe aber dem Gesetzeszweck der Entlastung zuwider"; Hervorhebung beibehalten. - Dieser von Graßhof wiedergegebene Gedankengang ist zirkulär. Auch wenn es zutreffen mag, daß die Begründung des Nicht-Vorliegens bestimmter Annahmegründe aufwendiger als die Begründung des Vorliegens bestimmter Ablehnungsgründe ist, erfolgte die Neufassung des $\$ 93 a$ doch im gleichen Zuge wie die des $\mathbb{9}$ 93d. Es handelt sich damit um ein vom Gesetzgeber selbst konstruiertes Problem, dem er durch eine andere Fassung der Änderung hätte entgehen können und jedenfalls bei einer erneuten Änderung entgehen kann.

34 Vgl. Graßhof (Fn. 33), $\mathbb{3}$ 93d Rn. 9.

35 F. Schemmer, $\mathbb{S} 93 \mathrm{~d}$, in: Umbach/Clemens/Dollinger, Bundesverfassungsgerichtsgesetz. Mitarbeiterkommentar und Handbuch, 2. Aufl. Heidelberg 2005, Rn. 6.

36 Schemmer (Fn. 35), $\mathbb{S} 93$ d Rn. 6. - Lenz/Hansel, Bundesverfassungsgerichtsgesetz. Handkommentar, Baden-Baden 2013, Rn. 10, fügen die Bemerkung hinzu, daß sich das Einstimmigkeitserfordernis nicht auf die Gründe der Nichtannahme erstrecke, da diese nicht Inhalt der Entscheidung seien; was freilich nur gilt, solange von einer Begründung im Beschluß abgesehen wird. Kischel wendet ein, daß ein Nichtübereinstimmen hinsichtlich der Gründe der Nichtannahme kaum die Mehrheit der Fälle betreffen dürfte (S. 213). In allen anderen Fällen würde ein Begründungsverzicht das unterliegende „materielle Problem nicht lösen, sondern es allenfalls überdecken und seine notwendige Lösung verzögern oder gar verhindern“ (S. 214). Kischel macht überdies den zustimmungswürdigen Vorschlag, das Erfordernis einer Begründung nicht als das einer einheitlichen Begründung zu verstehen. Vielmehr könnten auch unterschiedliche rechtliche Begründungen angegeben werden, solange sie zu dem gleichen Ergebnis führen (S. 215). 
aus Sicht der Kritiker, wünschenswerte Folge, daß mehr Verfassungsbeschwerden vom Senat, nicht von einer Kammer, entschieden würden.

Wenn Schemmer und Graßhof weiter darauf verweisen, daß $\mathbb{S} 93 \mathrm{~d}$ Abs. 1 S. 3 BVerfGG eine Begründung der Ablehnung durchaus zulasse, weswegen in einigen Fällen sogar regelmäßig Begründungen gegeben würden, ${ }^{37}$ so mag dies im Ergebnis die Bedenken noch erhöhen. Denn die Auswahl der Fälle, in denen ausnahmswei$\mathrm{se}^{38}$ Begründungen gegeben werden, wird nach nicht gesetzlich präskribierten Maßstäben allein vom Gericht getroffen, ${ }^{39}$ sie steht daher wiederum in dessen Belieben. Im Ergebnis streitet das Entlastungsargument zugunsten der Konzentration des Gerichts auf die übrigen, vielleicht verfassungsrechtlich bedeutenderen Verfahren, bedroht aber das Vertrauen der potentiellen, jedenfalls der nichtbegründet zurückgewiesenen Beschwerdeführer in die verfassungsrechtliche Judikatur.

\section{b) Verfassungsbeschwerde als außerordentlicher Rechtsbehelf}

Die Abwägung zwischen Konzentration und Vertrauen ist eine rechtspolitische Angelegenheit. Graßhof ergänzt ihre insofern rechtspolitische Rechtfertigungsstrategie der Entlastung um eine verfassungsrechtliche. Sie verweist auf den Vorbehalt des Annahmeverfahrens für die Verfassungsbeschwerde in Art. 94 Abs. 2 S. 2 GG, der zeitgleich mit der Verfassungsbeschwerde selbst in das Grundgesetz aufgenommen wurde..$^{40}$ Damit sei die Verfassungsbeschwerde von vornherein als außerordentlicher Rechtsbehelf gewährleistet, der sich als solcher „den Zwängen des,knappen Gutes‘ verfassungsgerichtlicher Rechtsprechungsressourcen zu unterwerfen“ habe. Dieses Argument der Außerordentlichkeit ist beachtlich; allerdings läßt sich dem verfassungsrechtlichen Vorbehalt eines Annahmeverfahrens nicht entnehmen, ob eine Nichtannahme nichtbegründet ergehen kann, soll oder gar muß. Insofern ist das verfassungsrechtliche Außerordentlichkeitsargument nicht eigenständig, es setzt vielmehr das rechtspolitische Entlastungsargument voraus. ${ }^{41}$

Graßhof wendet sich schließlich noch gegen den von Zuck vorgebrachten Vorwurf, der Bürger als Subjekt der Verfassungsbeschwerde dürfe nicht zum Objekt gerichtlicher Entlastung herabgewürdigt werden. Da dem Bürger nach Eingang seiner Verfassungsbeschwerde ein Merkblatt übersandt werde, in dem die gesetzlichen Annahmegründe erläutert, das Bedürfnis der Einstimmigkeit der Ablehnung erwähnt und

37 Vgl. Schemmer (Fn. 35), \$93d Rn. 6; Graßhof (Fn. 33), \93d Rn. 10 ff.

38 Nach Graßhof (Fn. 33), \93d Rn.6, ist die Inanspruchnahme der Befreiung von der Begründungspflicht der Regelfall; mit beispielhaften Zahlen aus der Jahresstatistik 2011 unterfüttern das Lenz/ Hansel (Fn. 36), \$ 93d Rn. 15. Der Erste Senat zeige sich „deutlich mitteilungsfreudiger“ als der recht verschlossene Zweite Senat. Diese Einschätzung unterstreichen die Zahlen aus dem Jahr 2013, vgl. noch unten, Fn. 65.

39 Auf diese gesetzlich eingeräumte Entscheidungsfreiheit der Kammern legen Lenz/Hansel (Fn. 36), $\int 93 \mathrm{~d}$ Rn. 12, besonderen Wert.

40 Vgl. oben, Fn. 32.

41 Umgekehrt wäre auch das Entlastungsargument nicht eigenständig, wenn die Nichtbegründung trotz eindeutiger Entlastung des Gerichts eindeutig verfassungswidrig wäre. Die Verfassungswidrigkeit aber ist jedenfalls nicht eindeutig gegeben, vgl. noch unten, Fn. 46 f. 
auf die mangelnde Begründungsbedürftigkeit hingewiesen werde, könne er, so Graßhof, sobald ihm der nichtbegründete Nichtannahmebeschluß zugegangen ist, nicht mehr ernstlich „den Eindruck von Beliebigkeit erhalten und sich als bloßes Objekt des Strebens des Bundesverfassungsgerichts nach Entlastung behandelt sehen ““. ${ }^{42} \mathrm{Ob}$ ein Formblatt den beschwerdeführenden Bürger in seiner Subjektqualität verstärkt, kann indes bezweifelt werden.

\section{Grundgesetzlicher Begründungszwang gerichtlicher Entscheidungen?}

Zuck jedenfalls beharrt in seinem Kommentar bis heute auf seinen Bedenken. Auch wenn er zugesteht, daß das Gericht durch nichtbegründete Nichtannahmen signifikant entlastet werde, stelle sich für den Bürger doch der „Eindruck willkürlicher Machtausübung “43 ein. Die verfassungsrechtliche Bewertung hänge nun allerdings nicht an diesem Eindruck, sondern daran, „ob es einen grundgesetzlichen Begründungszwang für gerichtliche Entscheidungen gibt" ${ }^{44} \mathrm{Im}$ Ergebnis schließt Zuck das aus; vor allem, weil es gegen die Entscheidung der Nichtannahme nach dem Grundgesetz keinen weiteren Rechtsweg gibt. ${ }^{45}$

Die Diskussion um einen grundgesetzlichen Begründungszwang soll hier nicht aufgenommen werden. Denn auch wer annimmt, daß die Möglichkeit der nichtbegründeten Nichtannahme bei allen Bedenken keinen Verstoß gegen das Grundgesetz

42 Für alles Graßhof (Fn. 33), $\$ 93 d$ Rn. 8.

43 R. Zuck, $\$ 93$ d Rn. 7, in: Bundesverfassungsgerichtsgesetz. Kommentar, begr. v. H. Lechner, fortg. v. R. Zuck, 6. Aufl. München 2011.

44 Zuck (Fn. 43), \$93d Rn. 7.

45 Vgl. Zuck (Fn. 43), \$93d Rn. 7. 


\section{bedeutet $^{46}$ - oder aber hinnimmt, daß sich eine Diagnose der Verfassungswidrig-} keit ${ }^{47}$ des $\$ 93 \mathrm{~d}$ Abs. 1 S. 3 BVerfGG entgegen der Einstimmigkeit von Gesetzgeber und Verfassungsgericht nicht durchsetzen wird -, ist damit nicht außerstande, Kritik zu üben. Der Maßstab der Kritik muß sich dann allerdings vom nationalen Verfassungsrecht entfernen, er verlagert sich auf internationalrechtliche, metarechtliche oder überrechtliche Kriterien. ${ }^{48}$

\section{Im folgenden Abschnitt soll, im Sinne einer metarechtlichen Kritik, Zucks Hinweis auf den Eindruck willkürlicher Machtausübung aufgenommen werden, den der Bür-}

46 Vgl. R. Rupprecht, Vorprüfung der Verfassungsbeschwerde, JZ 1970, S. 207-212, S. 212; Graßhof (Fn. 33), $\$ 93 d$ Rn. 8; P. Lange, Darlegungs- und Substantiierungspflichten im Verfassungsbeschwerdeverfahren, Baden-Baden 2012, S. 153; Lenz/Hansel (Fn. 36), $\$ 93 d$ Rn.11, unter Verweis auf BVerfGE 118, 212 (238). - In der angeführten Entscheidung geht das Bundesverfassungsgericht tatsächlich unter Verweis auf frühere Rechtsprechung davon aus, daß letztinstanzliche Entscheidungen nicht begründet werden müßten. Es ergänzt diesen Grundsatz aber um Erwägungen, nach denen eine Begründung gleichwohl „geboten“, gemeint ist wohl: verfassungsrechtlich geboten, sein könne: „Ungeachtet des Grundsatzes, wonach letztinstanzliche Entscheidungen nicht begründet werden müssen, ist in solchen Fällen angesichts des gerade in der Strafzumessung zum Ausdruck kommenden sozialethischen Unwerturteils über Tat und Täter eine Begründung geboten. Da mit der Kompetenz zur Strafzumessung durch das Revisionsgericht eine Durchbrechung der strafprozessualen Maximen der Unmittelbarkeit und Mündlichkeit sowie des Verfahrens in öffentlicher Hauptverhandlung einhergeht, die auch der Sicherung der Akzeptanz staatlichen Strafens dienen, kommt dem Begründungserfordernis eine kompensatorische Bedeutung zu. Eine Strafzumessungsentscheidung des Revisionsgerichts, die auf bislang im Verfahren nicht oder wesentlich anders gewichteten Umständen beruht, dies aber nicht erkennen lässt, würde die allgemeinen Grundsätze eines rechtsstaatlichen und transparenten Strafverfahrens nicht hinreichend beachten und brächte die Gefahr mit sich, dass sich der Angeklagte als Objekt staatlichen Handelns empfindet und die Akzeptanz der Entscheidung leidet". Gerade diese Argumente, die das Bundesverfassungsgericht hier für das Gebot der Begründung der Entscheidung des Revisionsgerichts heranzieht, sind aber unter denen, die die Kritiker gegen die Möglichkeit des Absehens von einer Begründung ins Feld führen.

47 Gegen die Verfassungsmäßigkeit des Annahmeverfahrens mit bis heute beachtlichen Argumenten schon Löw, Dauerproblem (Fn. 3), S. 941-944, und Sailer, Zwielicht (Fn. 3), S. 306-308, der vor allem auf die umfänglichen Ablehnungsgründe abstellt, mit denen das Vorprüfungsverfahren zu einem Vollprüfungsverfahren werde. - Für eine eingehende Herleitung eines verfassungsrechtlichen Begründungszwangs vgl. J. Lücke, Begründungszwang und Verfassung, Tübingen 1987, S. 37-124, der die einfachgesetzliche Ausnahme der eingeschränkten Begründungspflicht der Nichtannahme in der damals geltenden Fassung als „gar keine Begründung“ (S. 201) versteht, und diesen Begründungsausfall als einseitige Bevorzugung des Effizienzprinzips gegenüber einer ganzen Reihe anderer Verfassungsgüter betrachtet, was dem Grundsatz praktischer Konkordanz widerspreche (S. 203). Das BVerfGG sei daher insofern verfassungswidrig und damit auch rechtsstaatswidrig (S. 204). - Zum gleichen Ergebnis kommt U. Kischel, Die Begründung. Zur Erläuterung staatlicher Entscheidungen gegenüber dem Bürger, Tübingen 2003, der ebenfalls sehr tiefgehend die verfassungsrechtlichen Grundlagen der Begründung hoheitlicher Akte herausarbeitet (S. 63-142) und die Begründungspflicht als verfassungsrechtliches Prinzip im Sinne der Prinzipientheorie Robert Alexys einordnet (S. 171). Das gegenläufige Prinzip der Leistungsfähigkeit der Rechtsprechung (S. 188 f.) sei jedenfalls dann nachrangig, wenn sich aus der Begründung keine signifikante Mehrbelastung der Spruchkörper ergebe. Die Vorschrift des \93d Abs. 1 S. 3 BVerfGG sei deswegen und wegen der besonderen Bedeutung verfassungsgerichtlicher Entscheidungen „verfassungsrechtlich nicht haltbar“ (S. 208).

48 Zuck wählt die internationalrechtliche Perspektive. Er bemängelt eine Verletzung von Art. 6 Abs. 1 EMRK ,in den Fällen [...], bei denen der Entscheidung des BVerfG ein zivil- oder strafrechtliches Verfahren zugrunde gelegen hat", vgl. Zuck (Fn. 43), $\$ 93 d$ Rn. 7. Das Bundesverfassungsgericht (3. Kammer des Zweiten Senats) hat jüngst bestätigt, daß „Art. 6 EMRK in der Auslegung des Gerichtshofs [...] eine grundsätzliche Pflicht zur angemessenen Begründung gerichtlicher Entscheidungen zu entnehmen " sei. Damit sei allerdings unter bestimmten Umständen auch die Nichtbegründung einer letztinstanzlichen Entscheidung, hier die Verwerfung einer Revision durch den BGH nach $\mathbb{} 349$ Abs. 2 StPO, vereinbar, vgl. BVerfG NJW 2014, 2563 (2565). 
ger im Falle einer nichtbegründeten Nichtannahme seiner Verfassungsbeschwerde erhalten könne. Hierzu wird die Berechtigung des $\$ 93 \mathrm{~d}$ Abs. 1 S. 3 BVerfGG als Ausnahme von einer generellen Begründungspflicht anhand einiger Überlegungen aus diskurstheoretischer Perspektive hinterfragt.

\section{Begründung als Legitimation}

Die Diskurstheorie des Rechts behandelt das Verhältnis zwischen Begründen und Entscheiden im Recht. Entscheidungen können diskursiv, sei es im allgemeinen praktischen oder im juristischen Diskurs, begründet werden. Die Diskurstheorie bietet dazu die prozedurale Struktur. Weil die Diskurstheorie in erster Linie prozedurale Vorgaben enthält, ist kein Diskurs ergebnisdefinit. ${ }^{49}$ Das bedeutet, daß in jedem praktischen Diskurs zwei oder mehr gegensätzliche Verhaltensalternativen begründet werden könnten. Um angesichts dieser diskursiven Unsicherheit zu einem System von Handlungsanweisungen, einem Rechtssystem, zu gelangen, muß es entscheidungsbefugte Instanzen geben, die im Raum des diskursiv Möglichen eine Variante festsetzen. ${ }^{50}$

Von grundlegender Bedeutung für das gesamte System ist, daß die entscheidenden rechtlichen Instanzen nur im Raum des nach der Vorgabe der Verfassung und des auf ihr fußenden positiven Rechts ${ }^{51}$ diskursiv Möglichen agieren. Das diskursive Modell des Rechts verlangt deswegen, daß die Entscheidungen auf die Einhaltung dieses Rahmens überprüfbar sind. Für die Verwaltung übernahm diese Funktion in unserem Rechtssystem und seinen Vorläufern historisch das verwaltungsinterne Prüfverfahren in mehreren Instanzen, inzwischen erweitert oder abgelöst durch die Kontrolldienste einer unabhängigen Verwaltungsgerichtsbarkeit. Die Rechtsprechung kontrolliert sich ihrerseits, im Rahmen des Instanzenzuges, selbst. Rechtsprechung und Gesetzgeber schließlich werden, im System und im Rahmen der prozeduralen Vorgaben des Grundgesetzes, vom Bundesverfassungsgericht überprüft.

Da die entscheidenden Instanzen den juristischen Diskurs nicht nur qua Dezision beenden, sondern auch selber führen, kann im nachhinein nur anhand einer Begründung der Entscheidung durch die Entscheidenden überprüft werden, ob sie in diesem deliberativen Prozeß diskursive Fehler begangen haben. Ob der Gesetzgeber diesem Bedarf der Überprüfbarkeit hinreichend Rechnung trägt, indem er die Beratungen in den Parlamenten aufzeichnet und zugänglich macht, ist eine Frage, die sich zu unter-

49 Vgl. C. Bäcker, Begründen und Entscheiden. Kritik und Rekonstruktion der Alexyschen Diskurstheorie des Rechts, 2. Aufl., Baden-Baden 2012, S. 162-164.

50 Diese Offenheit des Modells für Entscheidungen im Raum des diskursiv Möglichen hat zur Konsequenz, daß namentlich der Richter einem Anspruch auf die einzig richtige Antwort nicht genügen kann. Wer den Richter dennoch diesen Anspruch erheben läßt, überfordert ihn, vgl. Bäcker, Begründen (Fn. 49), S. 326-334.

51 Es handelt sich dabei um die Bindung des juristischen Diskurses an Gesetz, Präjudiz und Dogmatik, näher Bäcker, Begründen (Fn. 49), S. 270-273. 
suchen lohnte. Hier soll es nur darum gehen, daß auch Gerichte ihre Entscheidungsgründe offenlegen müssen. Diesem diskurstheoretischen Erfordernis der Begründungstransparenz tragen die Prozeßordnungen grundsätzlich Rechnung.

\section{Entscheidungsgründe im Prozeßrecht}

Die Entscheidungsgründe sind nach den klassischen Prozeßordnungen im Grundsatz notwendige Bestandteile des Urteils. Im Zivilprozeßrecht regelt dies $₫ 313$ Abs. 1 ZPO. Dabei beschränken sich die Entscheidungsgründe allerdings, gem. $\$ 313$ Abs. 3 ZPO, auf „eine kurze Zusammenfassung der Erwägungen, auf denen die Entscheidung in tatsächlicher und rechtlicher Hinsicht beruht“. Gem. \$311 Abs. 3 ZPO ist diese kurze Zusammenfassung der Entscheidungsgründe im Rahmen der Urteilsverkündung nicht obligat. Die Entscheidungsgründe werden danach nur dann, „wenn es für angemessen erachtet wird, durch Vorlesung der Gründe oder durch mündliche Mitteilung des wesentlichen Inhalts verkündet“.

Eine besondere Ausnahme hält das Zivilprozeßrecht für die Begründung eines Beschlusses über die Beschwerde wegen der Nichtzulassung einer Revision in $\$ 544$ Abs. 4 S. 2 bereit. Hier heißt es: „Der Beschluss soll kurz begründet werden; von einer Begründung kann abgesehen werden, wenn sie nicht geeignet wäre, zur Klärung der Voraussetzungen beizutragen, unter denen eine Revision zuzulassen ist, oder wenn der Beschwerde stattgegeben wird“. In der Praxis hat sich diese Regel, nach der der zurückweisende Beschluß grundsätzlich zu begründen ist, dahin ausgeformt, daß „im Regelfall nur eine an den Gesetzestext angelehnte, formelhafte Begründung “ gegeben wird. ${ }^{52}$

Für das Bundesverfassungsgericht ist nicht nur diese Praxis verfassungsrechtlich unbedenklich, sondern auch der Verzicht auf jegliche Begründung einer letztinstanzlichen Entscheidung: ${ }^{53}$

In der Rechtsprechung des Bundesverfassungsgerichts ist geklärt, dass eine mit ordentlichen Rechtsmitteln nicht mehr anfechtbare letztinstanzliche gerichtliche Entscheidung von Verfassungs wegen regelmäßig keiner Begründung bedarf.

Das Zivilprozeßrecht enthält damit, aus der Sicht des Bundesverfassungsgerichts, regelmäßig dann verfassungsrechtlich überschießende Begründungspflichten, wenn es auch letztinstanzlichen Entscheidungen Begründungen abverlangt.

52 Vgl. W. Krüger, $\$ 544$ Rn. 27, in: ders./T. Rauscher (Hrsg.), Münchener Kommentar zur Zivilprozessordnung, Band 2, 4. Aufl. München 2012. Kritisch etwa H. Prütting, $\$ 544$ Rn. 39, in: B. Wieczorek (Begr.), R. A. Schütze (Hrsg.), Zivilprozeßordnung und Nebengesetze. Großkommentar, 3. Band, 1. Teilband, 3. Aufl., Berlin 2005, nach dem die Vorschrift als Begründungsverpflichtung zu lesen sei, der die in der Praxis üblichen „formelhaften Ergebnisklauseln“ nicht genügten.

53 BVerfG NJW 2011, 1497 (1497), 1. Kammer des Ersten Senats; unter Hinweis auf die ständige Rechtsprechung des Gerichts, u.a. auf BVerfGE 118, 212 (238) -vgl. dazu aber oben, Fn. 46. 
Im Strafprozeßrecht ist die Bekanntgabe der Urteilsgründe gem. $\mathbb{2 6 8}$ Abs. 2 StPO grundsätzlich ebenso ein notwendiger Bestandteil der Urteilsverkündung. $\$ 267$ StPO enthält dazu eine ganze Reihe spezifischer Festlegungen hinsichtlich der Form und des Inhalts der Urteilsgründe. Wird der Angeklagte verurteilt, so müssen gem. \$267 Abs. 1 StPO die Urteilsgründe „die für erwiesen erachteten Tatsachen angeben, in denen die gesetzlichen Merkmale der Straftat gefunden werden“. Ferner müssen gem. $\$ 267$ Abs. 3 StPO die „Gründe des Strafurteils [...] das zur Anwendung gebrachte Strafgesetz bezeichnen und die Umstände anführen, die für die Zumessung der Strafe bestimmend sind“. Im Falle eines Freispruchs des Angeklagten haben die Urteilsgründe gem. $\$ 267$ Abs. 5 StPO zu ergeben, „ob der Angeklagte für nicht überführt oder ob und aus welchen Gründen die für erwiesen angenommene Tat für nicht strafbar erachtet worden ist".

Rechtlich relevant im engeren Sinne werden die Entscheidungsgründe nur im Fall der Anfechtung einer richterlichen Entscheidung. Dies zeigt sich im Zivilprozeßrecht etwa daran, daß die Anfügung von Entscheidungsgründen gem. $\$ 313$ a Abs. 2 ZPO gänzlich entbehrlich ist, wenn „,beide Parteien auf Rechtsmittel gegen das Urteil verzichten“, sofern „das Urteil in dem Termin, in dem die mündliche Verhandlung geschlossen worden ist, verkündet" wird. Auch im Strafprozeßrecht sehen $\mathbb{2 6 7}$ Abs. 4 StPO für den Fall einer Verurteilung und $\$ 267$ Abs. 5 StPO für den Fall eines Freispruchs des Angeklagten erhebliche Erleichterungen hinsichtlich des Abfassens der Urteilsgründe vor, wenn alle zur Anfechtung Berechtigten auf Rechtsmittel verzichten. Im Falle des Freispruchs braucht sogar „nur angegeben zu werden, ob die dem Angeklagten zur Last gelegte Straftat aus tatsächlichen oder rechtlichen Gründen nicht festgestellt worden ist".

Würde sich die Bedeutung der Entscheidungsgründe tatsächlich in dieser Relevanz für Anfechtungen erschöpfen, wäre es in der Tat und im Sinne der angeführten Rechtsprechung des Bundesverfassungsgerichts überflüssig, letztinstanzliche Urteile überhaupt zu begründen. In der Konsequenz wären, zur Steigerung der Effizienz unserer höchsten Gerichte, nicht nur Verfassungsbeschwerden nichtbegründet nicht anzunehmen, sondern jede Entscheidung letztinstanzlicher Gerichte ohne Bekanntgabe einer Begründung zu fällen.

Nach den Prozeßrechtsordnungen sind jedoch im Regelfall auch letztinstanzliche Urteile, etwa des Bundesgerichtshofs, mit Entscheidungsgründen zu versehen. Für das Zivilprozeßrecht sei hier insbesondere die auf eine entgegenstehende Regel verweisende Ausnahme des $\$ 564$ S. 1 ZPO angeführt, wonach die Entscheidung „nicht begründet zu werden [braucht], soweit das Revisionsgericht Rügen von Verfahrensmängeln nicht für durchgreifend erachtet“. Für die Revisionsentscheidung im Strafprozeßrecht mag ein Hinweis auf $\$ 356$ StPO genügen, der für die Urteilsverkündung auf $\$ 268$ StPO verweist. Auch die Entscheidungen des Bundesverfassungsgerichts 
sind gem. $\$ 30$ Abs. 1 S. 2 BVerfGG zu begründen. Die amtlichen Entscheidungssammlungen enthalten Belege dafür, daß dieser Begründungspflicht gerade durch das Bundesverfassungsgericht zum Teil durchaus ausführlich entsprochen wird.

Wenn letztinstanzliche Begründungen von Verfassungs wegen entbehrlich sind, stellt sich die Frage nach dem Rechtsgrund für diese prozeßrechtlichen Begründungspflichten. Für letztinstanzliche Entscheidungen einfacher Gerichte ließe sich immerhin anführen, daß auch letztinstanzliche Urteile hinsichtlich ihrer VerfassungsmäBigkeit durch das Bundesverfassungsgericht überprüft werden können - und deswegen anhand gegebener Begründungen überprüfbar sein müssen. Für die Entscheidungen des Bundesverfassungsgerichts gilt dies aber nicht. ${ }^{54}$

\section{Begründungspflicht des Bundesverfassungsgerichts}

Die generelle Begründungspflicht des $\$ 30$ Abs. 1 S. 2 BVerfGG hat daher offenbar nicht den prozeßrechtlichen Hintergrund der Anfechtbarkeit. Sie hat einen metarechtlichen Horizont. Die Diskurstheorie kann diesen Horizont näher beschreiben. Sie bietet mit dem Begründungserfordernis für gerichtliche Entscheidungen eine Erklärung für die Begründungspflicht auch und gerade verfassungsgerichtlicher Entscheidungen an. Denn eine gerichtliche Entscheidung kann, im Sinne des dargelegten diskursiven Modells des Rechts, nur soweit legitim sein, wie sie, erstens, von der kompetenten, also nach den Regeln der Rechtsordnung zuständigen Instanz getroffen wurde, und, zweitens, diskursiv gerechtfertigt ist, also im Rahmen der diskursiven Möglichkeit relativ auf den zugrundeliegenden juristischen Diskurs geblieben ist.

Erst die Transparenz der Begründung durch die Angabe von Entscheidungsgründen ermöglicht es, diesen zweiten Legitimitätsmaßstab an die Entscheidung anzulegen. ${ }^{55}$ Die Entscheidungsgründe entfalten so eine legitimitätsbestimmende Funkti-

54 Allenfalls ließe sich anführen, daß der eine Senat von der Rechtsprechung des anderen Senats nur dann mit der verfassungsprozessualen Folge des $\mathbb{1 6}$ Abs. 1 BVerfGG abweichen kann, wenn die Entscheidungen mit Begründungen versehen werden.

55 Im Fokus der Diskurstheorie liegt allerdings der Rechtfertigungszusammenhang (context of justification), nicht der Entdeckungszusammenhang (context of discovery) juristischer Entscheidungen, vgl. R. Alexy, Juristische Interpretation, in: ders, Recht, Vernunft, Diskurs. Studien zur Rechtsphilosophie, Frankfurt/M. 1995, S. 71-92 (81). 
on. ${ }^{56}$ Die legitimatorischen Anforderungen ${ }^{57}$ an die Begründung richterlicher Entscheidungen verhalten sich dabei umgekehrt parallel zu den prozeßrechtlichen Möglichkeiten der Überprüfung. Grundsätzlich gilt: Je mehr kompetente Instanzen die gerichtliche Entscheidung auf ihre rechtliche Richtigkeit überprüfen können, umso geringer ist, aus der Sicht des diskursiven Modells des Rechts, der Bedarf einer legitimationsstiftenden Begründung. Nach diesem Grundsatz sind an nicht mehr rechtlich überprüfbare Entscheidungen nicht etwa keine, sondern die höchsten Begründungsanforderungen $\mathrm{zu}$ richten.

\section{a) Das Bundesverfassungsgericht als Letztinterpret}

Für die Entscheidungen des Bundesverfassungsgerichts ist es namentlich die herausgehobene Stellung in der Architektur des Rechtssystems, die diese Annahme stützt. ${ }^{58}$ Das Verfassungsgericht wacht über die Einhaltung der Verfassung. Es ist seine originäre Aufgabe, alle staatliche Gewalt am Maßstab des Verfassungsrechts zu kontrollieren - soweit das Verfassungsrecht dies vorsieht. In dieser Wächterrolle ist das Verfassungsgericht der Garant der bedeutendsten Voraussetzung des Gelingens jeden Verfassungsstaates: der Bindung aller staatlichen Gewalt an die Verfassung.

Als Wächter des Grundgesetzes kann das Bundesverfassungsgericht, ausweislich seiner ihm vor allem in Art. 93 GG übertragenen Entscheidungszuständigkeiten, wenn auch vorbehaltlich seiner Anrufung, die Akte der staatlichen Gewalt auf seine Verfassungsmäßigkeit hin überprüfen. Verwirklicht ist damit, nicht zuletzt, die Forderung des Art. 1 Abs. 3 GG, nach dem die Grundrechte des Grundgesetzes „Gesetzgebung, vollziehende Gewalt und Rechtsprechung als unmittelbar geltendes Recht“ binden.

Die Medaille der Verfassungsgerichtsbarkeit als Gewährleistung der effektiven Verfassungsbindung weist, auf ihrer anderen Seite, die Gefahr einer überschießenden

56 Die Annahme, Richtern komme eine Begründungspflicht zu, ist kein Alleinstellungsmerkmal der Diskurstheorie. Eine entsprechende These wird als selbstverständlich in der politischen Philosophie angenommen, vgl. nur J. Rawls, Politischer Liberalismus, übers. v. W. Hinsch, Frankfurt a.M. 2003, S. 316: „Verfassungsrichter in konstitutionellen Demokratien“ müssen „,ihre Urteile auf der Grundlage ihres Verständnisses der Verfassung und der relevanten Gesetze und Präjudizien begründen “; deutlicher im englischsprachigen Original, vgl. dens., Political Liberalism, New York: Cambridge University Press 1993, S. 216: „the justices have to explain and justify their decisions“. - Dieser Zusammenhang zwischen Begründung und Legitimation gerichtlicher Entscheidungen hat auch eine qualitative Dimension, vgl. V. A. da Silva, Deciding without Deliberating, ICON 11 (2013), S. 557-584 (559): „the legitimacy of judicial review is grounded (at least in part) in the argumentative quality of courts".

57 U. Di Fabio, Vom Recht, Recht zu sprechen: Die Legitimation des Bundesverfassungsgerichts, APuZ 2011, S. 3-7 (5), sieht in den Entscheidungen des Bundesverfassungsgerichts sogar „eine der großen Legitimationsquellen der Republik“. Ihrerseits bedarf diese Quelle der Legitimation umso mehr der Legitimität.

58 Ähnlich hat B.-O. Bryde, Transnationale Rechtsstaatlichkeit, Grundrechte und Solidarität. Durchsetzung und Verfahren, Festschrift für Renate Jäger, hrsg. v. C. Hohmann-Dennhardt, P. Masuch, M. Villiger, Kehl a.R. 2010, S. 65-73 (65), darauf hingewiesen, daß gerade auch letztentscheidende Gerichte der Kritik und Kontrolle bedürfen. 
verfassungsgerichtlichen Machtfülle auf. ${ }^{59}$ Dieser Versuchung der Macht müssen Verfassungsrichter widerstehen. ${ }^{60} \mathrm{Um}$ einen mit einer starken Verfassungsgerichtsbarkeit am Horizont stehenden Jurisdiktionsstaat zu vermeiden, in dem letzten Endes das Verfassungsgericht anhand seiner verfassungsrechtlich unanfechtbaren Interpretation des Verfassungsrechts über alle verfassungsrechtlichen Fragen letztwirksam entscheiden kann, muß es Spielräume des Gesetzgebers geben, in die die Zuständigkeit des Verfassungsgerichts nicht hineinreicht. Die Ausgestaltung dieser Spielräume ist Sache der Verfassung, in der die Zuständigkeiten und damit die kompetentiellen Grenzen aller staatlichen Gewalt einschließlich der ihres Wächters, des Verfassungsgerichts, zu regeln sind.

Das im einzelnen höchst komplizierte und dogmatisch wie rechtstheoretisch noch längst nicht durchmessene Verhältnis der Spielräume ${ }^{61}$ der staatlichen Gewalt auf der einen zu den Grenzen der verfassungsgerichtlichen Kontrolle auf der anderen Seite ist hier nicht im einzelnen nachzuvollziehen. Es muß der Hinweis genügen, daß dieses Verhältnis, im Holzschnitt, einfach zu beschreiben ist: Je enger das Verfassungsrecht die formellen und materiellen, also insbesondere kompetenzrechtlichen und grundrechtlichen, Grenzen für den Gesetzgeber zieht, desto weiter sind die verfassungsrechtlichen Spielräume für die zu seiner Kontrolle am Maßstab der Verfassung eingerichtete Verfassungsgerichtsbarkeit. Je enger, umgekehrt, die verfassungsrechtlichen Grenzen ${ }^{62}$ für das Verfassungsgericht gezogen sind, umso weiter sind die verfassungsrechtlichen Spielräume der gesetzgebenden Gewalt.

Soweit die Ausformung des Verhältnisses zwischen den staatlichen Gewalten, insbesondere der Legislative und ihrer Kontrolle durch das Verfassungsgericht, im Verfassungsrecht getroffen wird, unterliegt die Gestaltung des Spielraums der staatlichen Gewalt durch die Bestimmung der Grenzen der Verfassungsgerichtsbarkeit dem Verfassungsgeber. Dabei hat das Verfassungsgericht in der Architektur des Verfassungsstaates hinsichtlich des Ausgleichs zwischen Kontrolle und Spielraum den Trumpf der Letztinterpretation. Am Ende entscheidet das Verfassungsgericht darüber, welche Grenzen das Verfassungsrecht dem Verfassungsgericht zieht. Seine besondere Stellung ergibt sich also, entsprechend der Idee der Verfassungsgerichtsbarkeit, aus

59 Eingehend O. W. Lembcke, Hüter der Verfassung, Tübingen 2007, insb. im dritten Kapitel „Macht zur Selbstermächtigung “, S. 73-166.

60 Es ist nicht sicher, ob sie dieser Versuchung immer wiederstanden haben. Erst kürzlich hat der Verfassungsrichter R. Gaier in seiner abweichenden Meinung zu einem Plenarbeschluß der Gesamtheit seiner Richterkollegen vorgeworfen, das Gericht zu einem „verfassungsändernde[n] Ersatzgesetzgeber“ zu machen, BVerfGE 132, 1 (24).

61 Die Rekonstruktion dieser Spielräume stellt einen Schwerpunkt der gegenwärtigen Bemühungen in der Prinzipientheorie des Rechts dar, vgl. nur M. Klatt/J. Schmidt, Spielräume im Öffentlichen Recht. Zur Abwägungslehre der Prinzipientheorie, Tübingen 2010, S. 6 f.

62 Formelle Grenzen betreffen die Frage der Zuständigkeit des Verfassungsgerichts, einschließlich prozeduraler Voraussetzungen. Materielle Grenzen betreffen die Frage des anzuwendenden rechtlichen Maßstabs. 
dieser eigentümlichen Letztinterpretationshoheit auch bezüglich der Reichweite der eigenen Jurisdiktion.

In allen Fällen, in denen das Verfassungsgericht zur Entscheidung angerufen wird, hat es den Verfassungstext zu interpretieren und anzuwenden. Die Interpretationskompetenz des Bundesverfassungsgerichts ist dabei, etwa im Rahmen der Überprüfung von Gerichtsentscheidungen im Wege der Verfassungsbeschwerde, auf das Verfassungsrecht beschränkt. Die Auslegung einfachen Rechts bleibt daher im Grundsatz den Fachgerichten vorbehalten. ${ }^{63}$ Das Bundesverfassungsgericht macht dies seine Kompetenzen interpretierend - deutlich, wenn es sich ausdrücklich nicht als eine „Superrevisionsinstanz" versteht. ${ }^{64}$

Die hier nur aufscheinenden Fragen, die sich zum Verhältnis des Verfassungsgebers zum Verfassungsgericht stellen, müssen unbehandelt bleiben. $\mathrm{Zu}$ ihnen zählt etwa die Frage danach, inwiefern die trotz der Selbstbeschränkungen des Gerichts bestehende Einwirkung und Durchdringung des einfachen Rechts durch das Verfassungsrecht, namentlich durch die Grundrechte in Folge der Lüth-Entscheidung, zu einer Überkonstitutionalisierung führt. ${ }^{65}$ Für die vorliegende Untersuchung genügt es, zu wiederholen, daß das Bundesverfassungsgericht hinsichtlich der Interpretation des Verfassungstextes naturgemäß, jenseits einer Änderung des Verfassungstextes, nicht vom Verfassungsgeber beeinflußt werden kann. Die Determination des geltenden Verfassungsrechts ist Sache der Verfassungsgerichte. Die Verfassungsgerichte sind insofern die Letztinterpreten des Verfassungstextes; das Bundesverfassungsgericht ist der Letztinterpret des Grundgesetzes. ${ }^{66}$

\section{b) Begründungslosigkeit als Legitimitätsverlust}

Die Interpretationshoheit der Verfassungsgerichte ist gerade deswegen, und nur darum geht es hier, mit hohen Anforderungen an die verfassungsgerichtliche Praxis verknüpft, namentlich an die Begründung von Entscheidungen. Dem Bundesverfassungsgericht kommt, als Institution, deren Entscheidungen im Grundsatz rechtlich

63 Etwas anderes gilt ausnahmsweise dann, wenn das Bundesverfassungsgericht im Wege der abstrakten Normenkontrolle gem. Art. 93 Abs. 1 Nr. 2 GG „die Vereinbarkeit von Landesrecht mit sonstigem Bundesrechte" prüft.

64 Das Bundesverfassungsgericht ist keine „Superrevisionsinstanz“. Es ist nicht seine Aufgabe, die Rechtsprechung der zuständigen Fachgerichte bei der Auslegung des sogenannten „einfachen Rechts“ auf ihre Richtigkeit zu überprüfen oder gar zu vereinheitlichen. Es kann nach eigener Einschätzung im prozessualen Rahmen einer Verfassungsbeschwerde erst dann tätig werden, wenn die Entscheidung eines Gerichts Auslegungsfehler erkennen läßt, die auf einer grundsätzlich unrichtigen Anschauung von der Bedeutung und Reichweite eines Grundrechts beruhen oder wenn das Auslegungsergebnis sonst mit den Grundrechtsnormen nicht vereinbar ist, vgl. BVerfGE 18, 85 (92 f).

65 Vgl. für einen Eindruck der Bandbreite dieser Diskussion nur die Referate auf der 61. Staatsrechtslehrertagung zum ersten Beratungsgegenstand „Verfassungsrecht und einfaches Recht - Verfassungsgerichtsbarkeit und Fachgerichtsbarkeit" sowie die anschließend dokumentierte Aussprache.

66 Vgl. C. Bäcker, Wissenschaft als Amt. Das verfassungsrechtliche Hochschullehrerbeamtenrecht aus Art. 33 Abs. 5 GG i.V.m. Art. 5 Abs. 3 GG, AöR 135 (2010), S. 78-114 (107, dort Fn. 5). 
nicht mehr überprüft werden können, ${ }^{67}$ die Pflicht einer besonders eingehenden und auch für den Adressatenkreis verständlichen Begründung seiner Entscheidungen zu. Dabei ist es, trotz und wegen seiner rechtlich erhabenen Interpretationshoheit, an Regeln gebunden. Diese Regeln sind, auf einen Begriff gebracht, die des verfassungsrechtlichen Diskurses. Der Charakter dieser Bindung ist keine rechtliche Pflicht, soweit ihre Erfüllung rechtlich nicht überprüfbar ist. Es handelt sich um eine reine diskursive Pflicht, die dem Gericht sich selbst und seiner Aufgabe gegenüber zukommt. ${ }^{68}$

Eine Entscheidung des Bundesverfassungsgerichts muß also auch und insbesondere dann mit einer Begründung versehen sein, wenn und soweit sie rechtlich nicht mehr überprüfbar ist. Diskurstheoretischer Zweck der Begründung ist, um es zu wiederholen, die Ermöglichung der Überprüfung, ob die Entscheidung im Rahmen des juristisch Vertretbaren, also des juristisch diskursiv Möglichen geblieben ist. Ohne eine Begründung der Entscheidung wäre die Überprüfung nur auf hypothetische Weise möglich, indem die Entscheidung des Gerichts daraufhin überprüft wird, ob es eine vertretbare rechtliche Begründung für sie gibt. Die diskurstheoretische Begründungspflicht höchster Gerichte beschränkt sich jedoch nicht auf die Anforderung, daß die Entscheidungen begründbar sein müssen. Sie verlangt vielmehr die Ermöglichung der Überprüfung der Entscheidung auf ihre Vertretbarkeit; sie statuiert eine Art Bringschuld einer vertretbaren Begründung.

Die Ausnahme des $\$ 93 \mathrm{~d}$ Abs. 1 S. 3 BVerfGG, der es erlaubt, Verfassungsbeschwerden unbegründet und unanfechtbar nichtanzunehmen, bedeutet deswegen, diskurstheoretisch betrachtet, einen Legitimitätsverlust für das Gericht, wenn es von der Möglichkeit der nichtbegründeten Nichtannahme Gebrauch macht. ${ }^{69}$ Das Gericht entzieht sich hier seiner metarechtlichen Verantwortung. Davon bleibt unberührt, daß auch im Fall einer gegebenen, aber qualitativ schwachen Begründung ein Legi-

67 Das Verhältnis der drei höchsten Gerichte Europas, des Europäischen Gerichtshofes für Menschenrechte, des Europäischen Gerichtshofes und des Bundesverfassungsgerichtes zueinander ist höchst komplex und seit Jahren in der Diskussion. Die europäischen Gerichte, denen möglicherweise eine übernationalrechtliche Vorrangstellung gegenüber nationalen Gerichten zuzuerkennen ist, entscheiden jedenfalls nicht über die Auslegung des Grundgesetzes, sondern nur über die Auslegung der von ihnen jeweils zu überwachenden europäischen Rechtsordnungen. Für die vorliegende Untersuchung soll es daher genügen, das Bundesverfassungsgericht als letzte Instanz für die Auslegung und Anwendung des Grundgesetzes zu verstehen, was nicht kontrovers sein kann.

68 Insofern könnte man versucht sein, von einer diskursiven Obliegenheit zu sprechen. Der Begriff der Obliegenheit, der namentlich in der Dogmatik des allgemeinen Zivilrechts und des Versicherungsrechts näher ausgearbeitet wurde, läßt sich wie folgt näher fassen: Die „Befolgung der Obliegenheit [...] ist Gebot des eigenen Interesses, da der Belastete bei ihrer Verletzung einen Rechtsverlust oder rechtliche Nachteile erleidet“, C. Grüneberg, in: Palandt, 73. Aufl., München 2014, v. \$241 Rn. 13. Der Nachteil, den das Bundesverfassungsgericht im Falle der Verletzung dieser Begründungsobliegenheit zu befürchten hätte, bestünde in der Verminderung der Legitimität seiner Entscheidung bis hin zu einem möglichen Verlust im Falle einer ausbleibenden Begründung.

69 Dieser Legitimitätsverlust auf Seiten des Gerichts spiegelt sich, auf Seiten des Bürgers, in dem bemängelten „Schaden an staatsbürgerlicher Gesinnung“, Speckmann, Praxis (Fn. 3), S. 214. 
timationsdefizit vorläge. ${ }^{70}$ Der Verzicht auf jede Begründung ist jedoch nicht nur ein Qualitätsmangel, er ist kategorial.

\section{Ergebnis}

\$93d Abs. 1 S. 3 BVerfGG erlaubt den vollständigen Verzicht auf eine Begründung für die Nichtannahme einer Verfassungsbeschwerde, dem erhabensten Rechtsmittel des Bürgers. Zwar ist das Gericht rechtlich nicht daran gehindert, jede Nichtannahme zu begründen - doch sind die Versuchungen des Nichtbegründens angesichts der stetigen Flut eingehender Verfassungsbeschwerden offenbar zu groß. Das Bundesverfassungsgericht nutzt diese Möglichkeit der Entlastung jedenfalls intensiv. Der gerichtlichen Jahresstatistik 2013 zufolge begründete der Erste Senat lediglich ein Drittel seiner Nichtannahmen; der Zweite Senat versah kaum eine Nichtannahme mit einer Begründung. ${ }^{71}$ Diese Praxis genügt der metarechtlichen Begründungspflicht des Bundesverfassungsgerichts nicht.

Ist unserer Verfassungsgerichtsbarkeit zu wünschen, daß der Gesetzgeber ihr mit der Wiedereinführung ${ }^{72}$ einer Begründungspflicht für Nichtannahmen ${ }^{73}$ den diskurstheoretisch richtigen Weg weist? Dafür spricht, daß dem Gericht nur so die metarechtliche Unanfechtbarkeit zurückgegeben wäre, die seiner Stellung gebührt und die es durch sein Wirken verdient. ${ }^{74}$ Mit jeder zu begründenden Nichtannahme wäre das Gericht allerdings belasteter als zuvor.

Im Gegenzug müßte das Gericht also, um seine gegenwärtige Funktionsfähigkeit nicht einzuschränken oder gar zu gefährden, anderweit entlastet werden. In Betracht käme ein ganzes Bündel an Möglichkeiten, etwa eine Umstrukturierung des Gerichts

70 Für den Regelfall der begründeten Entscheidung ist der qualitative Legitimationszusammenhang von größter Bedeutung. Für eine Systematisierung einschlägiger Qualitätskriterien und ihre Anwendung auf die Begründungspraxis des brasilianischen Supreme Court vgl. da Silva, Deliberating (Fn. 56), die Fallstudie auf S. 567-584.

71 Für den Ersten Senat weist die Jahresstatistik 2013 von 5117 anhängigen Verfassungsbeschwerden aus den Jahren 2007-2013 3031 nicht angenommene Verfassungsbeschwerden aus, davon 1958 ohne Begründung, vgl. https:/www.bundesverfassungsgericht.de/organisation/gb2013/B-III-4.html. Für den Zweiten Senat weist die Jahresstatistik 2013 von 4731 anhängigen Verfahren aus den Jahren 2006-2013 2873 nichtangenommene Verfassungsbeschwerden aus, davon 2743 ohne Begründung, vgl. https://www.bundesverfassungsgericht.de/organisation/gb2013/C-III-4.html.

72 Vgl. zu den Vorfassungen oben, Fn.; einen Überblick gibt auch K. Graßhof (Fn. 33), $\mathbb{S} 93 d$ Rn. 5.

73 In die entgegengesetzte Richtung eines Begründungsverbots gehen Überlegungen von K. Schlaich/S. Korioth, Das Bundesverfassungsgericht. Stellung, Verfahren, Entscheidungen, 8. Aufl. München: Beck

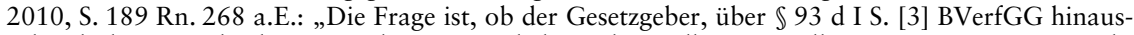
gehend, dem Gericht die Begründung unmöglich machen sollte. [...] Selbst wenn man so weit nicht gehen will, sollten die Begründungen von Nichtannahmeentscheidungen allenfalls auf den für die Ablehnung der Annahme maßgeblichen rechtlichen Gesichtspunkt hinweisen ".

74 Mit einem ähnlichen Aufruf beschloß schon im Jahr 1969 Vogel, Offensichtlich (Fn. 3), S. 801, seine Glosse: „Durch seine Rechtsprechung hat [das Bundesverfassungsgericht] sich viel Ansehen und Vertrauen erworben; es wäre für uns alle bedauerlich, wenn es sich dieses Vertrauen durch eine zu unbedenkliche Vorprüfungspraxis verscherzen würde". 
oder eine Verschärfung der Zulässigkeitsvoraussetzungen von Verfassungsbeschwerden. ${ }^{75}$ Jede einzelne dieser Möglichkeiten wäre jedoch eigens zu diskutieren.

75 Ein Bündel „rechtspolitische[r] Abhilfemaßnahmen“ gegen die Überlastung des Gerichts durch die Flut von Verfassungsbeschwerden diskutiert H. Posser, Zugangsschranken zum Bundesverfassungsgericht, in: F. J. Düwell (Hrsg.), Anwalt des Rechtsstaates. Festschrift für Dieter Posser zum 75. Geburtstag, Köln 1997, S. 331-359, darunter tiefgreifende Änderungen wie die Abschaffung der Verfassungsbeschwerde (344f.), die Einführung eines freien Annahmeverfahrens nach dem Vorbild des USamerikanischen Supreme Court (345-349) oder die Einführung von Vorprüfungskommissionen (355-358), aber auch weniger einschneidende Maßnahmen, wie die Einführung eines Anwaltszwanges (353 f.) oder Verschärfungen der Substantiierungslast (354 f.). Zum letztgenannten jüngst Lange, Substantiierungspflichten (Fn. 46), S. 183-188. 